Journal of Organometallic Chemistry, 290 (1985) 231-240

Elsevier Sequoia S.A., Lausanne - Printed in The Netherlands

\title{
METALLORGANISCHE LEWISSÄUREN
}

\section{XIX *. REAKTIONEN VON $\left(\pi-\mathrm{C}_{5} \mathrm{H}_{5}\right)(\mathrm{CO})_{2} \mathrm{LM}-\mathrm{X}\left(\mathrm{M}=\mathrm{Mo}, \mathrm{W} ; \mathrm{X}=\mathrm{FBF}_{3}, \mathrm{FPF}_{5}\right.$, $\mathrm{FAsF}_{5}, \mathrm{FSbF}_{5}$ ) MIT O-DONOREN}

\author{
KARLHEINZ SÜNKEL, GÜNTER URBAN und WOLFGANG BECK \\ Institut für Anorganische Chemie der Universität München, Meiserstr. I, 8000 München 2 (B.R.D.) \\ (Eingegangen den 1. Februar 1985)
}

\section{Summary}

The weakly coordinated anions $\mathrm{X}^{-}$in $\mathrm{Cp}(\mathrm{CO})_{2} \mathrm{LM}-\mathrm{X}(\mathrm{M}=\mathrm{Mo}, \mathrm{W} ; \mathrm{L}=\mathrm{CO}$, $\mathrm{PPh}_{3}, \mathrm{P}(\mathrm{OPh})_{3} ; \mathrm{X}=\mathrm{FBF}_{3}, \mathrm{FPF}_{5}, \mathrm{FAsF}_{5}$ and $\mathrm{FSbF}_{5}$ ) can easily be substituted by water, acetone, alcohol and diethyl ether to give the ionic compounds $\left[\mathrm{Cp}(\mathrm{CO})_{2} \mathrm{LML}^{\prime}\right]^{+} \mathrm{X}^{-}\left(\mathrm{L}^{\prime}=\mathrm{H}_{2} \mathrm{O}, \mathrm{Me}_{2} \mathrm{CO}, \mathrm{EtOH}, \mathrm{Me}_{2} \mathrm{CHOH}, \mathrm{Et}_{2} \mathrm{O}\right)$. These complexes easily hydrolyse and decompose in solution; the highest stabilities are observed with $\mathrm{M}=\mathrm{W}$ and $\mathrm{X}=\mathrm{SbF}_{6}$.

\section{Zusammenfassung}

Die schwach koordinierten Anionen $\mathrm{X}^{-}$in $\mathrm{Cp}(\mathrm{CO})_{2} \mathrm{LM}-\mathrm{X}(\mathrm{M}=\mathrm{Mo}, \mathrm{W} ; \mathrm{L}=\mathrm{CO}$, $\left.\mathrm{PPh}_{3}, \mathrm{P}(\mathrm{OPh})_{3} ; \mathrm{X}=\mathrm{FBF}_{3}, \mathrm{FPF}_{5}, \mathrm{FAsF}_{5}, \mathrm{FSbF}_{5}\right)$ lassen sich leicht durch Wasser, Alkohol, Aceton und Ether unter Bildung der ionogenen Verbindungen $\left[\mathrm{Cp}(\mathrm{CO})_{2} \mathrm{LML}^{\prime}\right]^{+} \mathrm{X}^{-}$substituieren $\left(\mathrm{L}^{\prime}=\mathrm{H}_{2} \mathrm{O}, \mathrm{Me}_{2} \mathrm{CO}, \mathrm{EtOH}, \mathrm{Me}_{2} \mathrm{CHOH}, \mathrm{Et}_{2} \mathrm{O}\right)$. Diese Komplexe sind gegen Hydrolyse labil und zersetzen sich in Lösung. Am stabilsten sind die Wolfram-Verbindungen mit $\mathrm{SbF}_{6}{ }^{-}$.

\section{Einführung}

Vor einiger Zeit haben wir gezeigt, dass sich das koordinierte Tetrafluoroborat in $\left(\pi-\mathrm{C}_{5} \mathrm{H}_{5}\right) \mathrm{Mo}(\mathrm{CO})_{3} \mathrm{FBF}_{3}$ leicht durch Aceton, Wasser und cyclische Ether nach Gl. 1 verdrängen lässt $[2,3]$ :

$$
\begin{aligned}
\mathrm{CpMo}(\mathrm{CO})_{3} \mathrm{FBF}_{3}+\mathrm{L} \rightarrow & {\left[\mathrm{CpMo}(\mathrm{CO})_{3} \mathrm{~L}\right]^{+} \mathrm{BF}_{4}{ }^{-} } \\
& \left(\mathrm{Cp}=\pi-\mathrm{C}_{5} \mathrm{H}_{5}\right) \\
& \left(\mathrm{L}=\mathrm{H}_{2} \mathrm{O}, \mathrm{Me}_{2} \mathrm{CO},\left(\mathrm{CH}_{2}\right)_{n} \mathrm{O} \text { mit } n=2,3,4\right)
\end{aligned}
$$

\footnotetext{
* XVIII. Mitteil. siehe Ref. 1.
} 
Solche Substitutionsreaktionen konnten wir auch mit verschiedenen $\mathrm{N}$ - und $\mathrm{P}$ Donoren sowie Olefinen beobachten [2,4]. Bei der Hydrolyse des $\mathrm{BF}_{4}{ }^{-}-\mathrm{K}_{\text {omplexes in }}$ Lösung entsteht u.a. das Trismetallaoxoniumsalz $\left\{\left[\mathrm{CpMo}(\mathrm{CO})_{2}\right]_{3} \mathrm{O}\right\}^{+} \mathrm{BF}_{4}^{-}$[5]. Ähnliche Hydrolysereaktionen wurden von Legzdins et al. für $\mathrm{Cp}_{2} \mathrm{Mo}(\mathrm{NO}) \mathrm{X} / \mathrm{AgBF}_{4}$ [6] und $\mathrm{CpCr}(\mathrm{NO})_{2} \mathrm{BF}_{4}$ [7] beschrieben.

Wir untersuchten nun allgemein das Reaktionsverhalten der metallorganischen Lewissäuren $\mathrm{CpM}(\mathrm{CO})_{2} \mathrm{LX}\left(\mathrm{M}=\mathrm{Mo}, \mathrm{W} ; \mathrm{L}=\mathrm{CO}, \mathrm{PPh}_{3}, \mathrm{P}(\mathrm{OPh})_{3} ; \mathrm{X}=\mathrm{FBF}_{3}\right.$, $\mathrm{FPF}_{5}, \mathrm{FAsF}_{5}$ und $\mathrm{FSbF}_{5}$ ) gegenüber Wasser und anderen harten Lewisbasen.

\section{Ergebnisse}

Durch Einwirkung von Wasserdampf auf die festen violetten Komplexe 1 konnten die roten Aquakomplexe 2 erhalten und anhand IR- und NMR-spektroskopischer Daten sowie analytisch charakterisiert werden.

$\mathrm{Cp}(\mathrm{CO})_{2} \mathrm{LMX}+\mathrm{L}^{\prime} \rightarrow\left[\mathrm{Cp}(\mathrm{CO})_{2} \mathrm{LML}^{\prime}\right]^{+} \mathrm{X}^{-}$

Bei Einwirkung von Wasser in Lösung werden Produktgemische erhalten. Eine Hydrolyse des $\mathrm{BF}_{4}$-Ions, wie mit $\mathrm{Mn}(\mathrm{CO})_{5} \mathrm{FBF}_{3}$ und $\mathrm{Re}(\mathrm{CO})_{5} \mathrm{FBF}_{3}$ [8] beschrieben, konnte nicht beobachtet werden. Auch mit le und If findet sich IR-spektroskopisch kein Hinweis für die Hydrolyse der Anionen [9]. Hingegen entsteht, wie auch in einigen anderen Fällen beobachtet [10,1], bei der Einwirkung von Wasser auf $\mathrm{CpW}(\mathrm{CO})_{3} \mathrm{FPF}_{5}$ (1g) kein Aquakomplex, sondern das $\mathrm{PO}_{2} \mathrm{~F}_{2}$-Ion (siehe experimenteller Teil). Durch Reaktion von äquimolaren Mengen oder mit einem geringen

TABELLE 1

AUSGANGSVERBINDUNGEN 1 UND PRODUKTE $2-5,\left[\mathrm{CpM}(\mathrm{CO})_{2} \mathrm{LL}^{\prime}\right]^{+} \mathrm{x}^{-}$

\begin{tabular}{|c|c|c|c|c|}
\hline la & Mo & $\mathrm{PPh}_{3}$ & - & $\mathrm{BF}_{4}$ \\
\hline $\mathbf{l b}$ & Mo & $\mathrm{P}(\mathrm{OPh})_{3}$ & - & $\mathrm{BF}_{4}$ \\
\hline le & W & $\mathrm{PPh}_{3}$ & - & $\mathrm{BF}_{4}$ \\
\hline ld & W & $\mathrm{P}(\mathrm{OPh})_{3}$ & - & $\mathrm{BF}_{4}$ \\
\hline $1 e$ & W & $\mathrm{CO}$ & & $\mathrm{AsF}_{6}$ \\
\hline If & $W$ & $\mathrm{CO}$ & - & $\mathrm{SbF}_{6}$ \\
\hline $\lg$ & W & $\mathrm{CO}$ & - & $\mathrm{PF}_{6}$ \\
\hline lh & W & $\mathrm{CO}$ & - & $\mathrm{BF}_{4}$ \\
\hline $\mathbf{2 a}$ & Mo & $\mathrm{PPh}_{3}$ & $\mathrm{H}_{2} \mathrm{O}$ & $\mathrm{BF}_{4}$ \\
\hline $2 b$ & Mo & $\mathrm{P}(\mathrm{OPh})_{3}$ & $\mathrm{H}_{2} \mathrm{O}$ & $\mathrm{BF}_{4}$ \\
\hline $2 c$ & $w$ & $\mathrm{PPh}_{3}$ & $\mathrm{H}_{2} \mathrm{O}$ & $\mathrm{BF}_{4}$ \\
\hline $2 d$ & W & $\mathrm{P}(\mathrm{OPh})_{3}$ & $\mathrm{H}_{2} \mathrm{O}$ & $\mathrm{BF}_{4}$ \\
\hline $2 \mathrm{e}$ & $\mathbf{w}$ & $\mathrm{CO}$ & $\mathrm{H}_{2} \mathrm{O}$ & $\mathrm{AsF}_{6}$ \\
\hline $2 \mathbf{f}$ & $w$ & $\mathrm{CO}$ & $\mathrm{H}_{2} \mathrm{O}$ & $\mathrm{SbF}_{6}$ \\
\hline $3 \mathbf{a}$ & Mo & $\mathrm{PPh}_{3}$ & $\mathrm{Me}_{2} \mathrm{CO}$ & $\mathrm{BF}_{4}$ \\
\hline $\mathbf{3 b}$ & $W$ & $\mathrm{PPh}_{3}$ & $\mathrm{Me}_{2} \mathrm{CO}$ & $\mathrm{BF}_{4}$ \\
\hline $3 \mathbf{c}$ & $w$ & $\mathrm{CO}$ & $\mathrm{Me}_{2} \mathrm{CO}$ & $\mathrm{BF}_{4}$ \\
\hline $\mathbf{4 a}$ & W & $\mathrm{CO}$ & $\mathrm{Me}_{2} \mathrm{CHOH}$ & $\mathrm{SbF}_{6}$ \\
\hline $4 b$ & W & $\mathrm{CO}$ & EtOH & $\mathrm{SbF}_{6}$ \\
\hline $5 \mathbf{a}$ & W & $\mathrm{CO}$ & $\mathrm{Et}_{2} \mathrm{O}$ & $\mathrm{PF}_{6}$ \\
\hline $\mathbf{5 b}$ & W & $\mathrm{CO}$ & $\mathrm{E}_{2} \mathrm{O}$ & $\mathrm{AsF}_{6}$ \\
\hline $5 c$ & W & $\mathrm{CO}$ & $\mathrm{Et}_{2} \mathrm{O}$ & $\mathrm{SbF}_{6}$ \\
\hline
\end{tabular}


TABELLE 2

IR- ${ }^{a}$ UND NMR-SPEKTROSKOPISCHE DATEN DER AQUAKOMPLEXE 2

\begin{tabular}{lllll}
\hline Verh & IR $\left(\mathrm{cm}^{-1}\right)$ & & $\begin{array}{l}\text { NMR } \\
\delta\left(\mathrm{C}_{5} H_{5}\right) \\
(\mathrm{ppm})\end{array}$ \\
\cline { 2 - 4 } & $\nu(\mathrm{CO})$ & $\nu(\mathrm{OH})$ & $\delta(\mathrm{OH})$ & $5.63^{c}$ \\
\hline 2a & $1982 \mathrm{~s}, 1893 \mathrm{~s}$ & $3390 \mathrm{~s}$ & $1670 \mathrm{w}$ & $5.36^{c}$ \\
2b & $2006 \mathrm{vs}, 1967 \mathrm{w}, 1933 \mathrm{~s}^{b}$ & $3360 \mathrm{w}$ & $1642 \mathrm{~m}, \mathrm{br}$ & \\
2e & $1958 \mathrm{vs}, 1870 \mathrm{vs}$ & & $1645 \mathrm{w}, \mathrm{br}$ & \\
2d & $1995 \mathrm{~s}, 1895 \mathrm{vs}$ & $3610 \mathrm{w}$ & & \\
& & $3520 \mathrm{w}$ & & $6.20^{d}$ \\
2e & $2062 \mathrm{~s}, 1977 \mathrm{vs}, 1950 \mathrm{vs}$ & $3350 \mathrm{~m}$ & $1613 \mathrm{~m}$ & $6.43^{e}$ \\
\hline
\end{tabular}

${ }^{a}$ In Nujol. ${ }^{b}$ In $\mathrm{CH}_{2} \mathrm{Cl}_{2} \cdot{ }^{c}$ In $\mathrm{CD}_{2} \mathrm{Cl}_{2}(\delta 5.33 \mathrm{ppm}){ }^{d}$ In $\mathrm{CD}_{3} \mathrm{NO}_{2}$ ( $\left.\delta 4.35 \mathrm{ppm}\right) .{ }^{e}$ In Aceton- $d_{6}(\delta 2.04$ ppm).

TABELLE 3

IR- ${ }^{a}$ UND NMR ${ }^{b}$-SPEKTROSKOPISCHE DATEN DER ACETONKOMPLEXE 3

\begin{tabular}{|c|c|c|c|c|c|}
\hline \multirow[t]{2}{*}{ Verb. } & \multicolumn{2}{|l|}{$\operatorname{IR}\left(\mathrm{cm}^{-1}\right)$} & \multicolumn{3}{|l|}{ NMR (ppm) } \\
\hline & $\overline{p(\mathrm{CO})}$ & $\nu(\mathrm{M}-\mathrm{O}=\mathrm{C})$ & $\delta\left(\mathrm{C}_{5} H_{5}\right)$ & $\delta\left(\mathrm{CH}_{3} \mathrm{CO}\right)$ & $\delta\left({ }^{31} \mathrm{P}\right){ }^{c}$ \\
\hline$c i s-3 \mathbf{a}$ & $1974 \mathrm{vs}, 1887 \mathrm{vs}$ & $1669 \mathrm{~s}$ & $5.74 \mathrm{~s}$ & $1.89 \mathrm{~s}$ & 50.7 \\
\hline trans-3a & $1993 \mathrm{~s}, 1901 \mathrm{vs}$ & $1657 \mathrm{~m}$ & $5.46 \mathrm{~d}(J 2 \mathrm{~Hz})$ & $2.37 \mathrm{~s}$ & 60.3 \\
\hline$c i s-\mathbf{3 b}$ & $1959 \mathrm{~s}, 1877 \mathrm{~s}$ & $1658 \mathrm{~m}$ & $5.91 \mathrm{~s}$ & $1.94 \mathrm{~s}$ & \\
\hline trans-3b & $1964 \mathrm{~s}, 1879 \mathrm{vs}$ & $1646 \mathrm{~s}$ & $5.55(J 2 \mathrm{~Hz})$ & $2.45 \mathrm{~s}$ & \\
\hline $3 c$ & $2050 \mathrm{~s}, 1930 \mathrm{vs}, \mathrm{br}$ & $1640 \mathrm{~m}, \mathrm{br}$ & $6.19 \mathrm{~s}$ & $2.43 \mathrm{~s}$ & \\
\hline
\end{tabular}

${ }^{a}$ In Nujol. ${ }^{b}$ In $\mathrm{CD}_{2} \mathrm{Cl}_{2}(\delta 5.33 \mathrm{ppm}) .{ }^{c} \mathrm{Rel} . \mathrm{H}_{3} \mathrm{PO}_{4}$.

TABELLE 4

IR- ${ }^{a}$ UND NMR-SPEKTROSKOPISCHE DATEN DER KOMPLEXE 4 UND 5 (L'= ROR')

\begin{tabular}{|c|c|c|c|c|c|c|c|c|c|c|}
\hline \multirow[t]{2}{*}{ Verb. } & \multirow[t]{2}{*}{$\mathbf{R}$} & \multirow[t]{2}{*}{$\mathbf{R}^{\prime}$} & \multirow[t]{2}{*}{$\mathrm{X}$} & \multicolumn{3}{|l|}{ IR $\left(\mathrm{cm}^{-1}\right)$} & \multicolumn{4}{|c|}{ NMR (ppm) } \\
\hline & & & & $\overline{p(\mathrm{CO})}$ & $\nu(\mathrm{OH})$ & $\overline{v(\mathrm{CO}-\mathrm{R})}$ & $\overline{\delta\left(\mathrm{C}_{5} H_{5}\right)}$ & $\delta(\mathrm{OCH})$ & $\delta\left(\mathrm{OCHCH}_{3}\right)$ & $\delta(\mathrm{OH})$ \\
\hline$\overline{4 a}$ & $\mathrm{H}$ & $\mathrm{Et}$ & $\mathrm{SbF}_{6}$ & $2058 \mathrm{~s}, 1962 \mathrm{vs}^{b}$ & $\begin{array}{l}3545 \mathrm{sh} \\
3460 \mathrm{sh} \\
3410 \mathrm{sh} \\
3350 \mathrm{~m}\end{array}$ & $1086 \mathrm{w}$ & $6.23^{\circ}$ & $3.91 \mathrm{dq}$ & $\begin{array}{l}1.16 \mathrm{t} \\
(J 7 \mathrm{~Hz})\end{array}$ & $\begin{array}{l}5.05 \mathrm{t} \\
(J 5 \mathrm{~Hz})\end{array}$ \\
\hline $4 b$ & $\mathbf{H}$ & $\mathrm{CHMe}_{2}$ & $\mathrm{SbF}_{6}$ & $2058 \mathrm{~s}, 1958 \mathrm{vs}$ & $3390 \mathrm{~m}$ & $1099 \mathrm{~m}$ & $6.22^{\circ}$ & $3.85 \mathrm{dh}$ & $\begin{array}{l}1.20 \mathrm{~d} \\
(J 6 \mathrm{~Hz})\end{array}$ & $\begin{array}{l}4.90 \mathrm{~d} \\
(J 7 \mathrm{~Hz})\end{array}$ \\
\hline $5 \mathbf{a}$ & Et & $\mathrm{Et}$ & $\mathbf{P F}_{6}$ & $2053 \mathrm{~s}, 1954 \mathrm{vs}$ & - & & $6.33^{\circ}$ & $\begin{array}{l}4.17 \mathrm{q} \\
(J 7 \mathrm{~Hz})\end{array}$ & $1.22 \mathrm{t}$ & - \\
\hline $\mathbf{5 b}$ & Et & $\mathrm{Et}$ & $\mathrm{AsF}_{6}$ & $2052 \mathrm{~s}, 1953 \mathrm{vs}$ & - & $1082 w$ & $6.34^{d}$ & $\begin{array}{l}4.24 \mathrm{q} \\
(J 7 \mathrm{~Hz})\end{array}$ & $1.22 \mathrm{t}$ & - \\
\hline $5 \mathbf{c}$ & Et & Et & $\mathrm{SbF}_{6}$ & $2061 \mathrm{~s}, 1962 \mathrm{vs}$ & - & $1086 w$ & $6.64^{d .}$ & $\begin{array}{l}4.20 \mathrm{q} \\
(J 7 \mathrm{~Hz})\end{array}$ & $1.15 \mathrm{t}$ & - \\
\hline
\end{tabular}

${ }^{a}$ In Nujol. ${ }^{b}$ In $\mathrm{CHCl}_{3} \cdot{ }^{c}$ In $\mathrm{CD}_{3} \mathrm{NO}_{2} \cdot{ }^{d}$ In Aceton- $d_{6 \cdot}{ }^{e}$ Bei $186 \mathrm{~K}$. dq: dublett von quartett dh: dublett von heptett q: quartett. 
Überschuss an Aceton mit 1a, 1c und 1h werden die mässig stabilen Komplexe 3 erhalten.

Die $\mathrm{PPh}_{3}$-haltigen Komplexe 3a und 3b fallen aus der Reaktionslösung überwiegend als trans-Isomere aus, die beim Wiederauflösen, wie aus den ${ }^{1} \mathrm{H}$ - und ${ }^{31} \mathrm{P}$-NMR-Spektren hervorgeht, vollständig zur cis-Form isomerisieren.

Isopropanol und Fthanol reagieren mit 1 fu den Komplexen 4a,b. Diethylether reagiert mit $\mathrm{CpW}(\mathrm{CO})_{3} \mathrm{FEF}_{5}(\mathbf{l e}-\mathbf{g})$ zu den in $\mathrm{CHCl}_{3}$ oder $\mathrm{CH}_{2} \mathrm{Cl}_{2}$ unlöslichen, kräftig gefärbten Ether-Komplexen 5a-c. Alle zersetzen sich in Lösung (Aceton oder Nitromethan) schon bei tiefer Temperatur, so dass die NMR-Spektren der reinen Verbindung nur bei $-60^{\circ} \mathrm{C}$ erhalten werden können.

Wird die Reaktion mit Diethylether bei Raumtemperatur durchgeführt, so entstehen die Tetracarbonyl-Komplexe $\left[\mathrm{CpW}(\mathrm{CO})_{4}\right]^{+} \mathrm{X}^{-}[11]$ als Hauptprodukte.

\section{Diskussion}

Als harte Lewisbasen bilden O-Donorliganden wie Wasser, Alkohole, Ether oder Ketone mit Metallen in niedrigen Oxidationsstufen in der Regel nur sehr labile Komplexe, die häufig als Ausgangsverbindungen in der präparativen metallorganischen Chemie dienen [12]. Beispiele finden sich v.a. mit den neutralen, ungesättigten Systemen $\mathrm{M}(\mathrm{CO})_{5}\left(\mathrm{M}=\mathrm{Cr}\right.$, Mo, W) [13] und $\mathrm{CpMn}(\mathrm{CO})_{2}$ [14] sowie den kationischen Lewissäuren " $\mathrm{M}(\mathrm{CO})_{5}{ }^{+}$" $(\mathrm{M}=\mathrm{Mn}, \mathrm{Re})[15]$ und " $\mathrm{CpFc}(\mathrm{CO})_{2}{ }^{+}$" [16], ausserdem mit einigen Metallen der Platingruppe [12,17].

Besonders mit den kationischen Komplexen treten häufig Folgereaktionen auf. So wirken Aqua-Komplexe als Brönstedsäuren auch gegen schwache Basen wie Ether, Wasser oder Aceton und geben zu Kondensationsreaktionen Anlass, wie sie auch aus der Ilauptgruppenchemie bekannt sind. Die Bildung von $\left\{\left[\mathrm{Cp}(\mathrm{CO})_{2} \mathrm{Mo}\right]_{3} \mathrm{O}\right\}^{+}[5]$, $\left\{\left[\mathrm{CpCr}(\mathrm{NO})_{2}\right]_{2}(\mathrm{OH})\right\}^{+}[7]$ oder $\left\{\left[\mathrm{Cp}_{2}\left(\mathrm{H}_{2} \mathrm{O}\right) \mathrm{Ti}\right]_{2} \mathrm{O}\right\}^{2+}[18]$ sei als Beispiel genannt. Ähnliche Kondensationsreaktionen wurden auch mit Alkoholen, besonders in Gegenwart stärkerer Basen beobachtet [19]. In Carbonylkomplexen tritt daneben noch der nucleophile Angriff am CO-Liganden hinzu, der zu Derivaten von Metallacarbonsäuren $\mathrm{L}_{n} \mathrm{M}-\mathrm{COOR}(\mathrm{R}=\mathrm{H}$ [20], alkyl [20c,21]) oder unter Weiterreaktion zu Hydriden führen kann [20b,21]. Hydride werden häufig auch bei der Reaktion mit Alkoholen, vor allem im Basischen, beobachtet [22]. Für Aceton werden im wesentlichen zwei Arten von Reaktionsweisen beschrieben: zum einen, die Koordination von Aceton über das O-Atom [2,8,12,15b,16a,16c], zum anderen, Aldolkondensation zum Diacetonalkohol bzw. zu Mesityloxid [10b,10c,23]. Letztere Reaktion, die auch mit Hauptgruppen-Lewissäuren beobachtet wird [24], kann entweder unter Verbleiben des Kondensationsproduktes in der Ligandensphäre [23] oder unter dessen Freisetzung verlaufen [25]. In den von uns untersuchten Reaktionen scheinen die Verbindungen 1 nur als koordinativ ungesättigte Systeme, nicht hingegen als Lewissäuren im Sinne der obengenannten Kondensationsreaktionen zu wirken.

Die Verbindungen 3a und 3h können als cis-/trans-Isomere vorliegen. Rei Reaktionsführung bei tiefer Temperatur fallen die trans-Isomeren als rote kristalline Niederschläge aus. Durch Wiederauflösen und Temperaturerhöhung auf $0^{\circ} \mathrm{C}$ und höher kann vollständige Umwandlung zum $c i s$-Isomeren erreicht werden [26]. Führt man die Reaktion bei $-10^{\circ} \mathrm{C}$ durch und/oder fällt man die Reaktionslösung mit Hexan, so werden Gemische erhalten, deren Zusammensetzungen u.a. auch von der 
Reaktionsdauer abhängen. Die Tendenz der Umwandlung, trans $\leftrightharpoons$ cis, ist somit der in $\mathrm{CpW}(\mathrm{CO})_{2} \mathrm{P}(\mathrm{OPh})_{3} \mathrm{FBF}_{3}$ beobachteten [27] cis $\rightarrow$ trans-Isomerisierung eines $\mathrm{BF}_{4}-$ Komplexes entgegengesetzt.

Während Komplexe von Metallen in niedrigen Oxidationsstufen mit cyclischen Ethern, v.a. THF, schon recht häufig beschrieben wurden $[2,12,20 \mathrm{~b}, 28]$, sind Verbindungen mit Diethylether als Ligand sehr selten charakterisiert und isoliert worden [13,17a,29]. Als Hinweis auf die schwachen Donoreigenschaften von $\mathrm{Et}_{2} \mathrm{O}$ mag gelten, dass sich die ansonsten sehr reaktiven Lewis-sauren Systeme $\mathrm{M}(\mathrm{CO})_{5} \mathrm{FBF}_{3}(\mathrm{M}=\mathrm{Mn}, \mathrm{Re})$ [30] und $\mathrm{CpFe}(\mathrm{CO})_{2} \mathrm{FBF}_{3}$ [31] aus entsprechenden Methylverbindungen und $\mathrm{HBF}_{4}$-etherat nach $\mathrm{Gl}$. 3 herstellen lassen, ohne dass ein $\mathrm{Ft}_{2} \mathrm{O}$-Komplex gefasst werden konnte:

$\mathrm{L}_{n} \mathrm{M}-\mathrm{CH}_{3}+\mathrm{HBF}_{4} \cdot \mathrm{OEt}_{2} \stackrel{\mathrm{OEt}_{2}}{\longrightarrow} \mathrm{L}_{n} \mathrm{MFBF}_{3}+\mathrm{CH}_{4}+\mathrm{OEt}_{2}$

Auch $\mathrm{CpCr}(\mathrm{NO})_{2} \mathrm{X}\left(\mathrm{X}=\mathrm{BF}_{4}\right.$ [7], $\mathrm{PF}_{6}$ [32]) scheinen nicht mit $\mathrm{Et}_{2} \mathrm{O}$ zu reagieren. Offensichtlich ist Diethylether ein schwächerer Donor als $\mathrm{BF}_{4}{ }^{-}$und $\mathrm{PF}_{6}{ }^{-}$. Die Beobachtung, dass $\operatorname{Re}(\mathrm{CO})_{5} \mathrm{CH}_{3}$ mit $\mathrm{HPF}_{6} \cdot \mathrm{OEt}_{2}$ nicht $\mathrm{zu} \operatorname{Re}(\mathrm{CO})_{5} \mathrm{FPF}_{5}$, sondern $\mathrm{zu}\left[(\mathrm{CO})_{5} \operatorname{ReFRe}(\mathrm{CO})_{5}\right]^{+} \mathrm{PF}_{6}{ }^{-}$reagiert [30], zeigt ausserdem, dass die Lewisbase $\mathrm{Et}_{2} \mathrm{O}$ erfolgreich mit der Base $\mathrm{Re}(\mathrm{CO})_{5} \mathrm{~F}$ um die Säure $\mathrm{PF}_{5}$ konkurriert. Die von uns dargestellten $\mathrm{Et}_{2} \mathrm{O}-\mathrm{Komplexe} 5$ sind hingegen unter trockenem Argon bei $-25^{\circ} \mathrm{C}$ mehrere Wochen unzersetzt stabil. Die Empfindlichkeit gegenüber Wasser nimmt in der Reihe $\mathrm{X}=\mathrm{SbF}_{6}, \mathrm{AsF}_{6}, \mathrm{PF}_{6}$ drastisch zu.

Die spektroskopischen Daten von 2-5 sind in den Tabellen 2-4 zusammengefasst. Die Aqua-Komplexe 2 zeigen i.a. breite $\nu(\mathrm{OH})$-Banden, was auf die Ausbildung von $\mathrm{H}$-Brücken zum Anion oder zu anderen $\mathrm{H}_{2} \mathrm{O}$-Molekülen erklärt werden kann. Ersteres scheint zumindest bei den Komplexen mit $\mathrm{X}=\mathrm{BF}_{4}$ der Fall zu scin, da die $\nu(\mathrm{B}-\mathrm{F})$-Banden entsprechend $C_{2 v}$-Symmetrie aufgespalten sind, wie schon früher beschrieben [2,5]. Im Gegensatz $\mathrm{zu}$ den kürzlich von Snow et al. beschriebenen Aqua-Komplexen $\left[\mathrm{M}(\mathrm{CO})_{3} \mathrm{~L}_{2}\left(\mathrm{H}_{2} \mathrm{O}\right)\right]^{+} \mathrm{X}^{-}\left(\mathrm{X}=\mathrm{BF}_{4}, \mathrm{AsF}_{6}\right)$ [33] zeigen unsere Komplexe 2 keine scharfe $\delta(\mathrm{OH})$-Bande bei $1600 \mathrm{~cm}^{-1}$, sondern ebenfalls nur breite Banden. Den OH-Protonen konnte im ${ }^{1} \mathrm{H}-\mathrm{NMR}-\mathrm{Spektrum}$ keine Resonanz eindeutig zugeordnet werden. Dagegen zeigen die OH-Protonen der AlkoholKomplexe 4 nicht nur die erwartete Intensität, sondern auch Kopplung zu den $\alpha$-ständigen CH-Protonen, was für die freien Alkohole im selben Lösungsmittel nicht beobachtet wird. Die $\nu(\mathrm{OH})$-Banden im IR-Spektrum sind deutlich schärfer als bei den Aqua-Komplexen, weshalb hier keine oder schwächere H-Brücken vorliegen dürften.

Die spektroskopischen Eigenschaften der Aceton-Komplexe 3 sind insofern bemerkenswert, als die Daten des Tricarbonyl-Komplexes $3 \mathbf{c}$ weitgehend denen der trans-Isomeren von $\mathbf{3 a}$ und $\mathbf{3 b}$ entsprechen, sich hingegen deutlich von denen der entsprechenden cis-Isomeren unterscheiden. Offensichtlich ist sowohl für die Methylresonanz im ${ }^{1}$ H-NMR-Spektrum als auch für die $\nu(\mathrm{CO}-\mathrm{M})$-Absorption im IR-Spektrum nur die Art der beiden cis-ständigen Liganden bestimmend. Die IR-Spektren der Ether-Komplexe 5 zeigen wenige charakteristische Banden, mit Vorbehalt lässt sich eine $\nu(\mathrm{C}-\mathrm{O}-\mathrm{M})$-Bande zuordnen (Tab. 4). Die ${ }^{1} \mathrm{H}-\mathrm{NMR}-$ Spektren zeigen hingegen eine deutliche Tieffeldverschiebung der dem koordinierenden Sauerstoff benachbarten Methylengruppe um etwa $1 \mathrm{ppm}$, während die Methylresonanz nur unwesentlich Tieffeld-verschoben erscheint. Dies wird auch in 


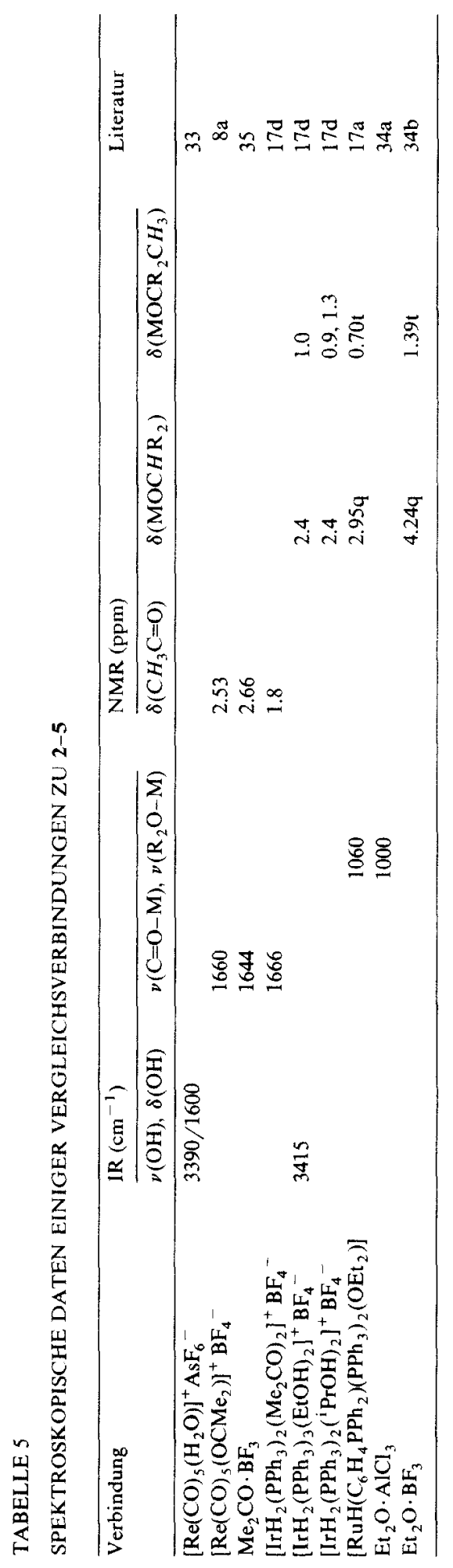


Etheraddukten mit Hauptgruppen-Lewissäuren beobachtet [34]. Die spektroskopischen Daten einiger Vergleichsverbindungen sind in Tab. 5 zusammengefasst.

Zusammenfassend lässt sich sagen, dass die metallorganischen Lewissäuren $\mathrm{CpW}(\mathrm{CO})_{3} \mathrm{FEF}_{5}(\mathrm{E}=\mathrm{As}, \mathrm{Sb})$ den Zugang zu isolierbaren Komplexen mit den in dieser Verbindungsklasse seltenen Liganden Alkohol und Diethylether eröffnen. Die relative Beständigkeit scheint auf die Schwerlöslichkeit der Verbindungen in den verwendeten Reaktionsmedien zurückzuführen sein. Alkohol- und Aqua-Komplexe wirken gegenüber schwachen Basen als Brönstedsäuren und bilden deshalb in Gegenwart von überschüssigem Liganden oder von Feuchtigkeit komplizierte Produktgemische.

\section{Experimenteller Teil}

Die Ausgangsverbindungen 1a und 1b wurden nach Lit. [4], 1c-1f wurden analog aus den entsprechenden Hydrido-Komplexen [36,37] und Tritylsalzen erhalten. Letztere wurden im Handel bezogen $\left(\mathrm{Ph}_{3} \mathrm{CBF}_{4}\right.$ : Fluka, Neu-Ulm, $\mathrm{Ph}_{3} \mathrm{CAsF}_{6}$ : Merck, Darmstadt, $\mathrm{Ph}_{3} \mathrm{CSbF}_{6}$ : Atlanta, Heidelberg) und gereinigt [4]. Alle Arbeiten erfolgen in Schlenk-Rohren in Argon-Atmosphäre in absolut trockenen, Argon-gesättigten Lösungsmitteln.

\section{Beispiel für die Darstellung der Komplexe 1}

Tricarbonyl(cyclopentadienyl)(hexafluoroantimonato)wolfram (If)

$1.25 \mathrm{~g} \mathrm{Ph}_{3} \mathrm{CSbF}_{6}\left(2.62 \mathrm{mmol}\right.$ ) werden in $20 \mathrm{ml} \mathrm{CHCl}_{3}$ aufgeschlämmt und auf $-55^{\circ} \mathrm{C}$ gekühlt. Etwa $0.67 \mathrm{~g}$ festes $\mathrm{CpW}(\mathrm{CO})_{3} \mathrm{H} \mathrm{[36]}(2.0 \mathrm{mmol})$ werden auf einmal zugegeben und die Suspension etwa $30 \mathrm{~min}$ gerührt, wobei eine deutliche Farbvertiefung nach purpur bis schwarz auftritt. Dann wird eine kleine Probe mittels einer Pipette entnommen und IR-spektroskopisch untersucht. Eine Bande bei ca. 1350 $\mathrm{cm}^{-1}$ zeigt die Anwesenheit von unumgesetztem $\mathrm{Ph}_{3} \mathrm{C}^{+} \mathrm{SbF}_{6}^{-}$an. Nun wird vorsichtig solange das feste Hydrid portionsweise zugegeben, bis nach jeweils $10 \mathrm{~min}$ Rühren das IR-Spektrum keine Bande bci $1350 \mathrm{~cm}^{-1}$ zeigt. Dann wird noch $10 \mathrm{~min}$ weitergerührt und zentrifugiert. Die überstehende violette Lösung wird in $20 \mathrm{ml}$ $-60^{\circ} \mathrm{C}$ kaltes Hexan gegossen und der entstandene Niederschlag durch Zentrifugieren isoliert. Beide Niederschläge werden bei $-60^{\circ} \mathrm{C}$ jeweils viermal mit je $10 \mathrm{ml}$ Hexan gewaschen und im Hochvakuum $8 \mathrm{~h}$ bei -25 bis $-10^{\circ} \mathrm{C}$ getrocknet. Es bleiben violette Pulver zurück. Ausbeute: 1.26 g (83\%). Wegen der extremen Empfindlichkeit von $1 f$ gegen Feuchtigkeit konnten keine befriedigenden C,H-Analysen erhalten werden.

Aqua(dicarbonyl)(cyclopentadienyl)(triphenylphosphan)molybdöntetrafluoroborat (2a)

Ein Schlenkrohr mit $0.25 \mathrm{~g}$ feinverteiltem $\mathrm{CpMo}(\mathrm{CO})_{2}\left(\mathrm{PPh}_{3}\right) \mathrm{FBF}_{3}$ wird zusammen mit einem Schälchen, gefüllt mit Wasser, in einem Exsiccator (der vorher evakuiert und mit Argon gefüllt wurde) $1 \mathrm{~h}$ im statischen Vakuum belassen. Das rote Pulver von $2 \mathrm{a}$ wird $8 \mathrm{~h}$ bei Raumtemperatur getrocknet. Ausbeute: quantitativ. (Gef.: C, 50.13; H, 3.46; $\mathrm{C}_{25} \mathrm{H}_{22} \mathrm{BF}_{4} \mathrm{MoO}_{3} \mathrm{P}$ ber.: C, 51.4; H, 3.80\%. Molmasse 584.15).

Die übrigen Aquakomplexe $\mathbf{2 b}-\mathbf{2 f}$ werden analog erhalten. Rührt man $2 \mathbf{f}$ einige Tage bei $-20^{\circ} \mathrm{C}$ mit Diethylether, so entsteht eine gelborange Lösung, die nach 
Abziehen des Ethers geringe Mengen eines Komplexes liefert, dessen IR-Spektrum in $\mathrm{CCl}_{4}$ Hinweise auf das Vorliegen von Carboxylat gibt $(2050 \mathrm{~m}, 1950$ s, 1908m; $1740 \mathrm{~m}, 1715 \mathrm{~m} \mathrm{~cm}^{-1}$ ).

Aceton(dicarbonyl)(cyclopentadienyl)(triphenylphosphan)molybdöntetrafluoroborat (3a)

$0.39 \mathrm{~g} \mathrm{Ph}_{3} \mathrm{CBF}_{4}(1.18 \mathrm{mmol})$ werden in $10 \mathrm{ml} \mathrm{CH}_{2} \mathrm{Cl}_{2}$ gelöst und bei $-40^{\circ} \mathrm{C} \mathrm{mit}$ festem $\mathrm{CpMo}(\mathrm{CO})_{2}\left(\mathrm{PPh}_{3}\right) \mathrm{H}$ [4] portionsweise versetzt, bis die übliche violette Farbe auftritt. Durch Zugabe von $8 \mathrm{ml}$ Hexan wird ein violetter Niederschlag gefällt, der durch Zentrifugieren isoliert und mit $30 \mathrm{ml}$ Hexan gewaschen wird. Dann wird bei $-40^{\circ} \mathrm{C} 10 \mathrm{ml} \mathrm{CH}_{2} \mathrm{Cl}_{2}$ und $0.1 \mathrm{ml} \mathrm{Aceton} \mathrm{(1.36} \mathrm{mmol)} \mathrm{zugegeben} \mathrm{und} \mathrm{drei} \mathrm{Tage} \mathrm{bei}$ $-25^{\circ} \mathrm{C}$ aufbewahrt. Ein Büschel von roten Kristallnadeln, trans-3a, wird von der überstehenden roten Lösung durch Abpipettieren getrennt, zweimal mit $10 \mathrm{ml}$ Hexan bei $-25^{\circ} \mathrm{C}$ gewaschen und anschliessend bei dieser Temperatur im Hochvakuum 8 h getrocknet. (Gef.: $\mathrm{C}, 52.72 ; \mathrm{H}, 4.34 ; \mathrm{C}_{28} \mathrm{H}_{26} \mathrm{BF}_{4} \mathrm{MoO}_{3} \mathrm{P}$ ber.: $\mathrm{C}, 53.88$; $\mathrm{H}, 4.20 \%$. Molmasse 624.24).

Durch Wiederauflösen in $20 \mathrm{ml} \mathrm{CH}_{2} \mathrm{Cl}_{2}$ und vierstündiges Rühren bei $+10^{\circ} \mathrm{C}$ kann cis-3a in Lösung erhalten werden. Durch Fällen und Waschen mit Hexan kann auch dieses Isomere sauber isoliert werden.

Aceton(dicarbonyl)(cyclopentadienyl)(triphenylphosphan)wolfram-tetrafluoroborat (3b)

$0.36 \mathrm{mmol} \mathrm{CpW}(\mathrm{CO})_{2}\left(\mathrm{PPh}_{7}\right) \mathrm{FBF}_{3}$ werden in $10 \mathrm{ml} \mathrm{CH}_{2} \mathrm{Cl}_{2}$ gelöst und bei $-30^{\circ} \mathrm{C}$ mit $5 \mathrm{ml}$ Aceton versetzt, wobei sofort ein gelber Niederschlag ausfällt, der abzentrifugiert wird. Man wäscht den Niederschlag von cis- und trans-3b viermal mit je $10 \mathrm{ml}$ Pentan und trocknet $5 \mathrm{~h}$ im Hochvakuum. (Gef.: C, 46.77; H, 4.46; $\mathrm{C}_{28} \mathrm{H}_{26} \mathrm{BF}_{4} \mathrm{O}_{3} \mathrm{PW}$ ber.: $\mathrm{C}, 47.22 ; \mathrm{H}, 3.68 \%$. Molmasse 712.14).

Aceton(tricarbonyl)(cyclopentadienyl)wolfram-tetrafluoroborat (3c)

$0.55 \mathrm{mmol} \mathrm{CpW}(\mathrm{CO})_{3} \mathrm{FBF}_{3}$ werden in $10 \mathrm{ml} \mathrm{CH}_{2} \mathrm{Cl}_{2}$ gelöst und bei $-30^{\circ} \mathrm{C}$ mit 3 $\mathrm{ml} \Lambda$ ceton versetzt. Nach einigen Minuten engt man im Vakuum ein und fällt mit 10 ml Pentan einen zunächst öligen roten Niederschlag aus. 3c wird viermal mit je 10 $\mathrm{ml}$ Pentan gewaschen und $5 \mathrm{~h}$ im Hochvakuum getrocknet. (Gef.: C, 26.90; H, 2.45; $\mathrm{C}_{11} \mathrm{H}_{11} \mathrm{BF}_{4} \mathrm{O}_{4} \mathrm{~W}$ ber.: $\mathrm{C}, 27.66 ; \mathrm{H}, 2.32 \%$. Molmasse 477.86)

Tricarbonyl(cyclopentadienyl)(ethanol)wolfram-hexafluoroantimonat (4b)

$0.475 \mathrm{~g} \mathrm{CpW} \mathrm{CO})_{3} \mathrm{FSbF}_{5}(0.84 \mathrm{mmol})$ werden in $10 \mathrm{ml} \mathrm{CHCl}_{3}$ bei $-45^{\circ} \mathrm{C}$ angelöst und mit $0.15 \mathrm{ml}$ Ethanol $(2.58 \mathrm{mmol})$ versetzt. Bald bildet sich ein rotes Öl. Die Reaktionslösung wird über Nacht bei $-25^{\circ} \mathrm{C}$ aufbewahrt und dann mit $20 \mathrm{ml}$ Hexan versetzt. Durch kräftiges Rühren und Schaben mit dem Spatel lässt sich das Öl verfestigen. Die überstehende Lösung wird abdekantiert, das Produkt wird nochmals mit $20 \mathrm{ml}$ Hexan bei $-30^{\circ} \mathrm{C}$ gewaschen. Nach erneutem Abdekantieren wird $4 \mathrm{~h}$ im Ölpumpenvakuum bei -35 bis $-5^{\circ} \mathrm{C}$ getrocknet. (Gef.: C, 18.2; $\mathrm{H}$, $1.86 ; \mathrm{C}_{10} \mathrm{H}_{11} \mathrm{~F}_{6} \mathrm{O}_{4}$ SbW ber.: C, 19.5; H, 1.80\%. Molmasse 614.79)

\section{Tricarhonyl(cyclopentadienyl)(isopropanol)wolfram-hexafluoroantimonat (4a)}

$0.215 \mathrm{~g} \mathrm{CpW}(\mathrm{CO})_{3} \mathrm{FSbF}_{5}(0.38 \mathrm{mmol})$ werden bei $-35^{\circ} \mathrm{C}$ mit $10 \mathrm{ml}$ Isopropanol versetzt. Nach wenigen Minuten entsteht ein purpurroter Niederschlag, der nach 15 min durch Zentrifugieren isoliert wird. 4 b wird $7 \mathrm{~h}$ im Ölpumpenvakuum bei -35 bis $-5^{\circ} \mathrm{C}$ getrocknet. (Gef.: C, 19.17; H, 2.21; $\mathrm{C}_{11} \mathrm{H}_{13} \mathrm{~F}_{6} \mathrm{O}_{4}$ SbW ber.: C, 21.0; $\mathrm{H}$, 2.08\%. Molmasse 628.81). 
Tricarbonyl(cyclopentadienyl)(diethylether)wolfram-hexafluorophosphat (5a)

$0.375 \mathrm{~g} \mathrm{Ph}_{3} \mathrm{CPF}_{6}(0.97 \mathrm{mmol})$ werden in $10 \mathrm{ml} \mathrm{CHCl}_{3}$ bei $-50^{\circ} \mathrm{C}$ suspendiert und mit $\mathrm{CpW}(\mathrm{CO})_{3} \mathrm{H}$ spatelweise bis zum ersten Auftreten einer violetten Färbung versetzt. Es wird noch 15 min weitergerührt und anhand des IR-Spektrums die Vollständigkeit der Reaktion überprüft (siehe oben). Bei $-38^{\circ} \mathrm{C}$ wird $0.1 \mathrm{ml} \mathrm{Et}_{2} \mathrm{O}$ (0.96 mmol) zugegeben und die Mischung über Nacht bei $-25^{\circ} \mathrm{C}$ aufbewahrt. Der entstandene violette Niederschlag, der in seiner Farbe nicht von der von 1g unterscheidbar ist, wird durch Zentrifugieren isoliert und zweimal mit je $10 \mathrm{ml}$ Hexan gewaschen und $6 \mathrm{~h}$ bei $-20^{\circ} \mathrm{C}$ im Hochvakuum getrocknet. (Gef.: C, 26.39; H, 1.99; $\mathrm{C}_{12} \mathrm{H}_{15} \mathrm{~F}_{6} \mathrm{O}_{4} \mathrm{PW}$ ber.: $\mathrm{C}, 26.11 ; \mathrm{H}, 2.74 \%$. Molmasse 552.06).

Tricarbonyl(cyclopentadienyl)(diethylether)wolfram-hexafluoroarsenat (5b)

$0.20 \mathrm{~g} \mathrm{CpW}(\mathrm{CO})_{3} \mathrm{FAsF}_{5}(0.38 \mathrm{mmol})$ werden in $20 \mathrm{ml} \mathrm{CHCl}_{3}$ bei $-50^{\circ} \mathrm{C}$ suspendiert und mit $1.0 \mathrm{ml} \mathrm{Et}{ }_{2} \mathrm{O}(9.6 \mathrm{mmol})$ versetzt, wobei sofort ein roter Niederschlag entsteht. Es wird noch 90 min weitergerührt, wobei die Temperatur auf maximal $-33^{\circ} \mathrm{C}$ ansteigt. Der Niederschlag wird durch Zentrifugieren isoliert, kurz bei Raumtemperatur und dann $6 \mathrm{~h}$ bei -30 bis $-10^{\circ} \mathrm{C}$ im Hochvakuum getrocknet. (Gef.: C, 24.05; H, 2.50; $\mathrm{C}_{12} \mathrm{H}_{15} \mathrm{AsF}_{6} \mathrm{O}_{4} \mathrm{~W}$ ber.: C, 24.18; H, 2.54\%. Molmasse 596.00).

Tricarbonyl(cyclopentadienyl)(diethylether)wolfram-hexafluoroantimonat (5c)

$0.575 \mathrm{~g} \mathrm{Ph}_{3} \mathrm{CSbF}_{6}(1.20 \mathrm{mmol})$ werden bei $-55^{\circ} \mathrm{C}$ in $20 \mathrm{ml} \mathrm{CHCl}_{3}$ suspendiert und mit $0.40 \mathrm{~g} \mathrm{CpW}(\mathrm{CO})_{3} \mathrm{H}(1.20 \mathrm{mmol})$ versetzt. Nach $30 \mathrm{~min}$ Rühren wird anhand des IR-Spektrums die Vollständigkeit der Reaktion überprüft. Bei $-49^{\circ} \mathrm{C}$ gibt man $1.0 \mathrm{ml} \mathrm{Et}{ }_{2} \mathrm{O}(9.6 \mathrm{mmol}) \mathrm{zu}$ und bewahrt die Lösung über Nacht bei $-25^{\circ} \mathrm{C}$ auf. Nach 5 h Rühren bei -50 bis $-25^{\circ} \mathrm{C}$ wird das Lösungsmittel am Hochvakuum bei $-20^{\circ} \mathrm{C}$ abgezogen. Der dunkelrote Rückstand wird zweimal bei $-50^{\circ} \mathrm{C}$ mit je $20 \mathrm{ml}$ Hexan gewaschen und $6 \mathrm{~h}$ bei -30 bis $-15^{\circ} \mathrm{C}$ im Hochvakuum getrocknet. (Gef.: C, 21.2; H, 2.12, $\mathrm{C}_{12} \mathrm{H}_{15} \mathrm{~F}_{6} \mathrm{O}_{4}$ SbW ber.: C, 22.4; H, 2.35\%. Molmasse 642.84)

Reaktion von $\mathrm{CpW}(\mathrm{CO})_{3} \mathrm{FPF}_{5}$ (Ig) mit feuchtem $\mathrm{CH}_{2} \mathrm{Cl}_{2}$

Etwa $0.2 \mathrm{~g} 1 \mathrm{~g}$ werden mit $10 \mathrm{ml}$ handelsüblichem, feuchtem $\mathrm{CH}_{2} \mathrm{Cl}_{2}$ versetzt und über Nacht bei $-25^{\circ} \mathrm{C}$ aufbewahrt. Es wird zentrifugiert und das dunkelrote Pulver $5 \mathrm{~h}$ bei Raumtemperatur im Hochvakuum getrocknet. IR: $\nu(\mathrm{CO}) 2048 \mathrm{~s}, 1950 \mathrm{vs}$, br, $\nu\left(\mathrm{PO}_{2} \mathrm{~F}_{2}\right) 1268 \mathrm{w}, 1218 \mathrm{vw}, 1148 \mathrm{~m}, 1060 \mathrm{~m}, 843 \mathrm{vs}, \mathrm{br}, 557 \mathrm{vs} \mathrm{cm}^{-1}$.

\section{Dank}

Der Deutschen Forschungsgemeinschaft und dem Fonds der Chemischen Industrie gilt unser herzlicher Dank für die Förderung unserer Arbeiten.

\section{Literatur}

1 H. Bauer, U. Nagel und W. Beck, J. Organomet. Chem., 290 (1985) 219.

2 W. Beck und K. Schloter, Z. Naturforsch. B, 33 (1978) 1214.

3 K. Schloter und W. Beck, Z. Naturforsch. B, 35 (1980) 985.

4 K. Sünkel, H. Ernst und W. Beck, Z. Naturforsch. B, 36 (1981) 474.

5 K. Schloter, U. Nagel und W. Beck, Chem. Ber., 113 (1980) 3775.

6 P. Legzdins und C.R. Nurse, Inorg. Chem., 21 (1982) 3110. 
7 P. Legzdins, D.T. Martin, C.R. Nurse und B. Wassink, Organometallics, 2 (1983) 1238.

8 K. Raab, U. Nagel und W. Beck, Z. Naturforsch. B, 38 (1983) 1466; K. Raab, B. Olgemöller, K. Schloter und W. Beck, J. Organomet. Chem., 214 (1981) 81; K. Raab und W. Beck. Chem. Ber., im Druck.

9 Vgl. L. Kolditz und B. Nussbücker Z. Anorg. Allg. Chem., 337 (1965) 191.

10 Vgl. z.B. (a) P. Legzdins und J.C. Oxley, Inorg. Chem., 23 (1984) 1053; (b) M.A. Bennett. T.W. Matheson, G.B. Robertson, W.L. Steffen und T.W. Turney, J. Chem. Soc., Chem. Commun., (1979) 32; (c) S.J. Thompson, P.M. Bailey, C. White and P.M. Maitlis, Angew. Chem. 88 (1976) 506; (d) F.L. Wimmer und M.R. Snow, Aust. J. Chem., 31 (1978) 267.

11 K. Schloter, Dissertation, Universität München 1979.

12 J.A. Davies und F.R. Hartley, Chem. Rev., 81 (1981) 79.

13 I.W. Stolz, G.R. Dobson und R.K. Sheline, Inorg. Chem., 2 (1963) 323.

14 (a) W. Strohmeier und D. v. Hobe, Z. Phys. Chem., 34 (1962) 393; (b) W. Strohmeier und J.F. , Guttenberger, Chem. Ber., 97 (1964) 1256; (c) H. Berke, J. Organomet. Chem., 185 (1980) 75.

15 (a) M.R. Snow und F.L. Wimmer, Inorg. Chim. Acta, 44 (1980) L189; (b) M. Oltmanns und R. Mews, Z. Naturforsch. B, 35 (1980) 1324.

16 (a) W.E. Williams und F.J. Lalor, J. Chem. Soc., Dalton Trans., (1973) 1329; (b) D.L. Reger, C.J. Coleman und P.J. McElligott, J. Organomet. Chem., 171 (1979) 73; (c) E.K.G. Schmidt und C.H. Thiel, J. Organomet. Chem., 209 (1981) 373; (d) P.F. Boyle und K.M. Nicholas. J. Organomet. Chem.. 114 (1976) 307.

17 (a) D.J. Cole-Hamilton und G. Wilkinson, J. Chem. Soc., Dalton Trans., (1977) 797; (b) J.A. Davies, F.R. Hartley und S.G. Murray, J. Chem. Soc., Dalton Trans., (1980) 2246; (c) J. Dehand, M. Pfeffer und M. Zinsius, J. Organomet. Chem., 118 (1976) C62; (d) R.H. Crabtree, P.C. Demou, D. Eden, J.M. Mihelcic, C.A. Parnell, J.M. Quirk und G.E. Morris, J. Amer. Chem. Soc., 104 (1982) 6994.

18 U. Thewalt und G. Schleussner, Angew. Chem., 90 (1978) 559.

19 Vgl. z.B. W. Schulze, K. Weidenhammer und M.L. Ziegler, Angew. Chem., 91 (1979) 432.

20 (a) D.H. Gibson, K. Owens und T.-S. Ong, J. Amer. Chem. Soc., 106 (1984) 1125; (b) J.R. Sweet und W.A.G. Graham, Organometallics, 1 (1982) 982; (c) N. Grice, S.C. Kao und R. Pettit, I. Amer. Chem Soc., 101 (1979) 1627.

21 (a) L. Malatesta, M. Angoletta und G. Caglio, J. Chem. Soc. A, (1970) 1836; (b) T. Kruck und M. Noack, Chem. Ber., 97 (1964) 1693; (c) W. Hieber und V. Frey, Chem. Ber., 99 (1966) 2614; (d) H.C. Clark und W.J. Jacobs, Inorg. Chem., 9 (1970) 1229.

22 (a) H.D. Kaesz und R.B. Saillant, Chem. Rev., 72 (1972) 231; (b) B.N. Chaudret, D.J. Cole-Hamilton, R.S. Nohr und G. Wilkinson, J. Chem. Soc. Dalton Trans., (1977) 1546.

23 (a) R.D. Gillard, B.T. Heaton und M.F. Pilbrow, J. Chem. Soc. A, (1970) 353; (b) C. White. S.J. Thompson und P.M. Maitlis, J. Organomet. Chem., 134 (1977) 319.

24 R. Kane, J. Prakt. Chem., 15 (1838) 129.

25 M. Di Vaira, P. Stoppioni und F. Mani, J. Organomet. Chem., 247 (1983) 95.

26 Die Zuordnung der Signale erfolgt nach dem Kriterium, dass im ${ }^{1}$ H-NMR-Spektrum die Kopplungskonstanten $J\left(\mathrm{P}, \mathrm{C}_{5} \mathrm{H}_{5}\right)$ in Verbindungen dieses Typs für trans-grösser als für cis-Isomcre sind: J.W. Faller und A.S. Anderson, J. Amer. Chem. Soc., 91 (1969) 1550.

27 K. Sünkel, G. Urban und W. Beck, J. Organomet. Chem., 252 (1983) 187.

28 (a) D.L. Reger und C. Colcman, J. Organomet. Chem., 131 (1977) 153; (b) J.K. Hoyano, C.J. May und W.A.G. Graham, Inorg. Chem., 21 (1982) 3095; (c) D. Sellmann und E. Kleinschmidt, J. Organomet. Chem., 140 (1977) 211.

29 (a) E. Lindner, S. Trad, S. Hoehne und H.-II. Oetjen, Z. Naturforsch. B, 34 (1979) 1203; (b) H. Behrens, E. Lindner und G. Lehnert, J. Organomet. Chem., 22 (1970) 439; (c) E. Klei und J.H. Teuben, J. Organomet. Chem., 188 (1980) 97; (d) J.M. Manriquez, P.I. Fagan, T.J. Marks, S.H. Vollmer, C. Secaur-Day und V.W. Day, J. Amer. Chem. Soc., 101 (1979) 5075.

30 K. Raab und W. Beck, Chem. Ber., im Druck.

31 A. Cutler, persönliche Mitteilung.

32 F.J. Regina und A. Wojcicki, Inorg. Chem., 19 (1980) 3803.

33 E. Horn und M.R. Snow, Aust. J. Chem., 37 (1984) 1375.

34 (a) R.L. Richards und A. Thompson, J. Chem. Soc., A (1967) 1244; (b) R.A. Craig und R.E. Richards, Trans. Faraday Soc., 59 (1963) 1962.

35 P.N. Gates und E.F. Mooney, J. Inorg. Nucl. Chem., 30 (1968) 839.

36 R.B. King. Organometallic Syntheses, Vol. 1, Academic Press, New York 1965, S. 156.

37 P. Kalck, R. Pince, R. Poilblanc und J. Roussel, J. Organomet. Chem., 24 (1970) 445. 